\title{
Dimensionality dependence of the self-interaction correction in the local-density approximation to density functional theory
}

\author{
I. Nagy ${ }^{1,2}$ and P. M. Echenique ${ }^{2,3}$ \\ ${ }^{1}$ Department of Theoretical Physics, Institute of Physics, Technical University of Budapest, H-1521 Budapest, Hungary \\ ${ }^{2}$ Donostia International Physics Center (DIPC), P. Manuel de Lardizabal 4, 20018 San Sebastián, Spain \\ ${ }^{3}$ Departamento de Física de Materiales and Centro Mixto CSIC-UPV/EHU, Facultad de Ciencias Químicas, Universidad del País Vasco, \\ Apdo. 1072, 20080 Sebastián, Spain
}

(Received 30 September 2009; revised manuscript received 15 December 2009; published 26 March 2010)

\begin{abstract}
The local-density approximation (LDA) to density functional theory is not self-interaction free. Motivated by this intellectual challenge, and the possible practical importance of strong electron-correlation in a Wignertype model, the capability of LDA is investigated for a two-dimensional electron system in the low-density limit. It is found for this essentially single-electron limit that the performance of LDA is slightly better in two dimension than in the equivalent three-dimensional problem treated earlier in Phys. Rev. B 18, 6506 (1978). An a priori explanation of this fact is given in terms of the different characters of potential fields generated by normalized charge distributions in these dimensions.
\end{abstract}

DOI: 10.1103/PhysRevB.81.113109

PACS number(s): 71.10.-w, 71.45.Gm, 71.15.Mb

$$
\epsilon_{b b}=\frac{1}{2} \frac{D}{D-1} \frac{R^{2 D-1}}{r_{s}^{2 D}} \frac{D}{(2 D-1) ! !}\left(\frac{\Gamma(D)}{\Gamma[(D+1) / 2]}\right)^{2}
$$

In the terminology of the review article ${ }^{1}$ of Walter Kohn is governed by the radical effect of the electron-electron interaction $v(r)=e^{2} / r$. The most accurate numerical calculations for such systems are performed by quantum Monte Carlo simulations ${ }^{4,5}$ in three and two dimensions. The output energies per particle of these calculations are used to constrain an input form to the local-density approximation (LDA) for the exchange-correlation energy at low densities. A well-constrained form for this many-body term is vital in the practical a posteriori implementation of the Kohn-Sham orbital method as applied to various interacting manyelectron systems.

While the exact density functional for the ground-state energy is self-interaction free, the practical method based on LDA is not. The elimination of the self-interaction is, therefore, an important issue. ${ }^{6}$ In this Brief Report, we shall investigate the two-dimensional (2D) self-interaction problem for the low-density, i.e., Wigner lattice limit since this is one of the limits on which an interpolation procedure to design an input exchange-correlation energy per particle in LDA is based. The real advantage of using the low-density limit is that in this important limit one has an essentially singleelectron problem. Due to this fact, it is easier to identify the contribution of the self-interaction error, as was explicitly pointed out earlier ${ }^{7}$ for the three-dimensional (3D) case. Following this logic, we start by a short review of energetics at low densities. We will use Hartree atomic units, $\hbar=m_{e}=e^{2}=1$, in our comparative study.

In a classical, point charge in the continuum, Wigner-Seitz modeling we take an electron to the center of the chargecompensating (rigid) background with a certain radius $R$. There are two terms contributing to a variational lattice $(L)$ energy $\epsilon_{L}(D)$ in this ${ }^{8}$ modeling. The Coulomb interaction energy $\left[\epsilon_{e b}(D)\right]$ of the pointlike electron with the positive jellium background, and the background self-energy $\left[\epsilon_{b b}(D)\right]$ are

$$
\epsilon_{e b}=-\frac{D}{D-1} \frac{R^{D-1}}{r_{s}^{D}},
$$

where $r_{s}$ is the Wigner-Seitz radius. ${ }_{-}^{8}$ After a variational procedure, we get the $R=r_{s}$ and $R=(\sqrt{\pi} / 2) r_{s}$ values in 3D and $2 \mathrm{D}$, respectively, at which the classical lattice-energies $\epsilon_{L}(D) \equiv \epsilon_{e b}(D)+\epsilon_{b b}(D)$ per particle are optimal. In a quantum-mechanical treatment, we have an oscillator $(o s)$ contribution of $\epsilon_{o s}(D)=\left[D /\left(2 r_{s}^{3 / 2}\right)\right]$ to the above sum of two terms in the lattice energy.

We stress the point that, in 3D, the $R=r_{s}$ derived here via a variational method fits completely to the remarkable theorem established ${ }^{9}$ by Lieb and Narnhofer. According to this theorem, the 3D lattice energy is bounded from below by the value, $\epsilon_{L}(3 D)=-0.9 / r_{s}$, obtained for it via the standard (and simple) Wigner-Seitz picture.

In order to model the strong electron-electron interaction in the fluid phase, we can use Wigner's original (as noted by Kirkwood $)^{10}$ picture in which a slow electron is surrounded by an electrostatically driven hole. Taking this normalized hole as a positive charge-distribution with an $r_{s}$ radius, as the energetically optimal limiting result due to electron-electron interaction, we get

$$
\epsilon_{x c}(D)=\frac{1}{2}\left[-\frac{D}{D-1} \frac{1}{r_{s}}\right]
$$

which is one-half the term given by Eq. (1) at $R=r_{s}$. At low densities the quantum-mechanical kinetic energy contributions $\left(\sim r_{s}^{-2}\right)$ to $\epsilon_{x c}(D)$ become negligible.

It is important to note that in his first work on the 3D prototype many-body system, Wigner used the above $\epsilon_{x c}(3 D)=-0.75 / r_{s}$ expression and subtracted from it the Hartree-Fock, i.e., exchange-only, $\epsilon_{x}(3 D)$ value in order to define a correlation energy. ${ }^{1,2,10}$ One has 


$$
\epsilon_{x}(D)=-\frac{2}{\pi} p_{F} \frac{D}{D^{2}-1},
$$

in terms ${ }^{8}$ of the Fermi wave-number $p_{F}$. From this, we obtain $\epsilon_{x}(3 D) \cong-0.46 / r_{s}$ and $\epsilon_{x}(2 D) \cong-0.60 / r_{s}$.

Next we illustrate the applicability of the above-outlined simple energetics via an estimation for transition between different ${ }^{11}$ phases in our model system. To this estimation we use the $\epsilon_{x c}(D)=\epsilon_{L}(D)+\left[D /\left(2 r_{s}^{3 / 2}\right)\right]$ constraint, i.e., we neglect the kinetic term which is higher-order in the inverse density parameter. With the above variational values for the $R$ radius, we get $r_{s}(3 D)=100$ and $r_{s}(2 D)=9(2 \sqrt{\pi}-3)^{-2} \cong 30$ for the transition values. These are, within few percent, in good agreement with predictions $\left[r_{s}(3 D)=106 \pm 1\right.$, and $\left.r_{s}(2 D)=31 \pm 1\right]$ based on quantum Monte Carlo ${ }^{4,5}$ simulations. However, one should keep in mind the remark of Anderson $^{11}$ about an interpolation ${ }^{2,6}$ procedure. He pointed out the delicate nature of the commonly applied interpolation between different phases.

Notice, in passing, that the numerical values of the ratio of $\epsilon_{L}(D) / \epsilon_{x}(D)$ are close (from below) to two independently of $D$, as was emphasized ${ }^{12}$ in a recent analysis on bounds in LDA. Considering the common scaling $\left[\sim\left(1 / r_{s}\right)\right]$ of the leading terms in the energies of the many-body system at low densities, one has to use the $\epsilon_{x c}^{(L D A)}(D)=-\beta / r_{s}$ form to design properly a local input-construction to numerical applications. $^{7,13}$

In the Wigner lattice, a pointlike electron sees only the normalized compensating charge density of the rigid background. Thus, in an LDA treatment, the spurious selfinteraction term, which is one-half the integrated ${ }^{6,7}$ electrostatic interaction of a normalized electronic chargedistribution with the potential field generated by itself, must cancel the energy-contribution calculated via a local (input) exchange-correlation term $\left[\epsilon_{x c}^{(L D A)}(r)\right]$. Earlier, insightful work gave, based on this robust global constraint, the $\beta(3 D) \cong 0.675(<0.75<0.9)$ value as the optimal (maximum) one in the $3 \mathrm{D}$ case. $^{7}$

At this point we introduce our main a priori statement on the dimensionality-dependence of deviations in LDA from the bounded exchange-correlations. Our statement rests on the following simple observation. The ratio $\left[\epsilon_{b b}(2 D) / \epsilon_{b b}(3 D)\right]>1$ at $R=r_{s}$. This heralds, in the light of the above-outlined cancellation-constraint, than in 2D-LDA with arbitrary (normalized) charge distributions we will get a closer agreement with bounds on exchange-correlation than in the $3 \mathrm{D}$ case.

So, how close can we go in 2D-LDA to the established rigorous bounds in the investigated low-density limit? Our quantitative estimations are based on three forms of the electronic charge distribution. The first of them

$$
n_{H}(r)=\frac{2 \alpha^{2}}{\pi} e^{-2 \alpha r}
$$

corresponds to a standard hydrogenic probability distribution function. The second is a Gaussian

$$
n_{G}(r)=\frac{\alpha}{\pi} e^{-\alpha r^{2}}
$$

Finally, in order to allow a more tunable (via $\mu>0$ ) representation, a power function is introduced

$$
n_{P}(r)=\frac{\alpha}{\pi} \frac{\mu}{\left(1+\alpha r^{2}\right)^{\mu+1}} .
$$

The cancellation constraint to estimate an optimal $\beta(2 D)$ value to $2 \mathrm{D}-\mathrm{LDA}$ is formulated as follows:

$$
\beta \int d r r[\pi n(r)]^{1 / 2} n(r) \equiv \frac{1}{2} \int \operatorname{drr} \varphi(r) n(r),
$$

where $\varphi(r)$ is the potential field generated by the given electronic charge distribution $n(r)$ itself.

The calculation of this field is, however, more complicated $^{14}$ in 2D real-space than in 3D. We illustrate this with the $n_{H}(r)$ distribution, with which we have

$$
r \varphi_{H}(r)=2 u^{2}\left[I_{0}(u) K_{1}(u)-I_{1}(u) K_{0}(u)\right],
$$

in terms ${ }^{15}$ of a shorthand $u \equiv \alpha r / 2$. The asymptotic form at $r \rightarrow \infty$ is $r \varphi_{H}(r) \rightarrow\left[1+3 /\left(2 \alpha^{2} r^{2}\right)\right]$, which signals that the decay is slow in 2D. In $3 \mathrm{D}$, the corresponding (hydrogenic) limit is, as is well-known from textbooks on quantum mechanics, unity to an exponential accuracy. The observed long-range differences in the fields generated by charge distributions are behind the enhanced values of the self-energy integrations, Eq. (8), in 2D.

In practice, we performed the integration on the left-hand side of Eq. (8) in real space, while the right-hand side (RH) is evaluated by using the convolution (faltung) theorem. Applying this theorem, we easily get

$$
(R H)=\frac{1}{2} \int_{0}^{\infty} d q q\left[n(q) \frac{1}{q} n(q)\right],
$$

where $n(q)$ is the Fourier-Hankel transform of $n(r)$. In the most complicated [power $(P)]$ case the result is

$$
\beta_{P}(\mu)=\frac{\pi(3 \mu+1)}{4^{(\mu+1)} \mu^{5 / 2}} \frac{\Gamma(2 \mu+1 / 2) \Gamma(\mu+1 / 2)}{[\Gamma(\mu)]^{3}} .
$$

One gets $\beta_{P}(\mu=1 / 2)=5 /(4 \sqrt{2}) \simeq 0.88$. From Eq. (11) $\beta_{P}(\mu \longrightarrow 0)=(\pi / 2)^{2} \sqrt{\mu}$ and $\beta_{P}(\mu \rightarrow \infty)=(3 / 4) \sqrt{\pi / 2}$. This last value is equal to the one obtained in the Gaussian case, where $\beta_{G}(2 D)=(3 / 4) \sqrt{\pi / 2}$ also. With $n_{H}(r)$ the (very similar) result is $\beta_{H}(2 D)=27 \pi /(64 \sqrt{2})$.

Therefore, quite independently of the applied models, we have a $-0.94 / r_{s}$ maximum-type estimation for the exchangecorrelation energy per particle within 2D-LDA of density functional theory in the low-density limit. It is, therefore, the best representation for this important limit which is free from the spurious self-interaction in a local treatment. This result differs only a little from $\epsilon_{x c}(2 D)=-1 / r_{s}$ obtained for the homogeneous gas at low densities of the $2 \mathrm{D}$ system. The ratio of $\left[\left(-0.94 / r_{s}\right) / \epsilon_{L}\left(R=r_{s}\right)\right]$ is, on the other hand, about 0.82 in $2 \mathrm{D}$. The corresponding ratio in the $3 \mathrm{D}$ case $^{7}$ has an about 0.75 value, due to $\beta(3 D) \cong 0.675$. This informative ratio also signals a stronger deviation in $3 \mathrm{D}$. 
As a future direction we propose to investigate, following an earlier insightful work ${ }^{16}$ on the hydrogen atom in $3 \mathrm{D}$, a gradient-corrected correlation functional in the Wigner limit where one is dealing with isolated electrons. In such a treatment ${ }^{16}$ for this important limit, the correlation potential of the functional must cancel the $\phi(r)$ potential of Eq. (8) locally. Since the $\phi(r)$ Coulomb potential depends on the applied single-particle orbital, as a comparison of Eq. (9) with the Gaussian case

$$
r \varphi_{G}(r)=\sqrt{2 \pi z} I_{0}(z) e^{-z}
$$

clearly shows $\left[z=\alpha r^{2} / 2\right]$, the results for the correlation potential will be orbital-dependent. We note finally that the many-electron self-interaction error, beyond the one-electron self-interaction problem discussed in our comparative study, has also a broad interest in constructions of universally useful approximate functionals. ${ }^{17}$

In conclusion, there is, as we explained a priori in this short work, a better performance of the LDA in 2D than in $3 \mathrm{D}$ at the low-density limit, where we have an essentially single-electron problem. The necessary self-interaction correction on an input many-body term to LDA in DFT is, therefore, smaller in 2D at this limit. Immediate applications of the result found in our study could be a modification of the LDA-based input exchange-correlation in thicknessdependent modeling ${ }^{13}$ of an electron layer, and in the fundamental problem of bound states ${ }^{18}$ around a negative point charge in a $2 \mathrm{D}$ electron system at low densities. In the attractive, so-called interaction strength interpolation modeling ${ }^{19,20}$ of exchange and correlation, the input at strong coupling rests on the details of the Wigner limit, as well.

\section{ACKNOWLEDGMENTS}

We thank N. W. Ashcroft, N. H. March, and E. Zaremba for useful discussions on various aspects of the problem investigated here, and A. Howie for careful reading of the manuscript. Financial supports from the Departamento de Educación del Gobierno Vasco, and the Spanish Ministerio de Ciencia y Technología (MCyT) (Grant No. FIS200766711-C01-01) are acknowledged.
${ }^{1}$ W. Kohn, Rev. Mod. Phys. 71, S59 (1999).

${ }^{2}$ E. Wigner, Phys. Rev. 46, 1002 (1934); Trans. Faraday Soc. 34, 678 (1938).

${ }^{3}$ G. D. Mahan, Many-Particle Physics (Plenum, New York, 1990), pp. 405-410.

${ }^{4}$ N. D. Drummond, Z. Radnai, J. R. Trail, M. D. Towler, and R. J. Needs, Phys. Rev. B 69, 085116 (2004).

${ }^{5}$ N. D. Drummond and R. J. Needs, Phys. Rev. Lett. 102, 126402 (2009).

${ }^{6}$ J. P. Perdew and A. Zunger, Phys. Rev. B 23, 5048 (1981) especially its paragraph IV.

${ }^{7}$ H. B. Shore, E. Zaremba, J. H. Rose, and L. Sander, Phys. Rev. B 18, 6506 (1978).

${ }^{8}$ I. Nagy, Phys. Rev. B 60, 4404 (1999).

${ }^{9}$ E. Lieb and H. Narnhofer, J. Stat. Phys. 12, 291 (1975); K. Moulopoulos and N. W. Ashcroft, Phys. Rev. Lett. 69, 2555 (1992).

${ }^{10}$ R. D. Cowan and J. G. Kirkwood, Phys. Rev. 111, 1460 (1958).

${ }^{11}$ P. W. Anderson, Basic Notions of Condensed Matter Physics
(Benjamin, London, 1984), pp. 128-129.

${ }^{12}$ E. Räsänen, S. Pittalis, K. Capelle, and C. R. Proetto, Phys. Rev. Lett. 102, 206406 (2009).

${ }^{13}$ D. Jost and M. W. C. Dharma-wardana, Phys. Rev. B 72, 195315 (2005).

${ }^{14}$ S. H. Patil, Eur. J. Phys. 29, 517 (2008).

${ }^{15}$ I. Nagy, M. J. Puska, and N. Zabala, Phys. Rev. B 74, 115411 (2006).

${ }^{16}$ P. M. W. Gill and J. A. Pople, Phys. Rev. A 47, 2383 (1993); D. J. Tozer, ibid. 56, 2726 (1997).

${ }^{17}$ P. Mori-Sanchez, A. J. Cohen, and W. Yang, J. Chem. Phys. 125, 201102 (2006); A. Ruzsinszky, J. P. Perdew, G. I. Csonka, O. A. Vydrov, and G. E. Scuseria, ibid. 126, 104102 (2007) and references therein.

${ }^{18}$ I. Nagy, N. Zabala, and P. M. Echenique, New J. Phys. 11, 063012 (2009).

${ }^{19}$ M. Seidl, J. P. Perdew, and M. Levy, Phys. Rev. A 59, 51 (1999).

${ }^{20}$ Z. F. Liu and K. Burke, Phys. Rev. A 79, 064503 (2009). 